\title{
Conservation of angular momentum and the inverse Faraday effect
}

\author{
S. R. Woodford* \\ Theorie-1, Institut für Festkörperforschung, Forschungszentrum Jülich, D-52425 Jülich, Germany
}

(Received 9 June 2008; revised manuscript received 3 June 2009; published 26 June 2009)

\begin{abstract}
Precession of magnetization via the inverse Faraday effect is investigated with a view of determining the fundamental limit on the precession speed. Such a limit could have important consequences for ultrafast magnetic switching. The angular momentum required for precession is shown to be supplied by the light. This indicates that there is no fundamental obstruction to magnetization reversal on the time scale of a laser pulse provided that a material with a sufficiently strong magneto-optical response can be found.
\end{abstract}

DOI: 10.1103/PhysRevB.79.212412

PACS number(s): 75.60.Jk, 75.40.Gb, 78.20.Ls, 85.70.Li

The ability to control magnetization on a subpicosecond time scale is growing in importance as the speed of electronic devices increases. The current generation of technology employs magnetic fields to induce magnetization dynamics. However, due to the difficulty of creating ultrashort magnetic pulses and the recent discovery that magnetic switching by strong magnetic fields can be unpredictable, ${ }^{1}$ alternative techniques of controlling magnetization are under intense investigation. ${ }^{2-17}$

Optical methods ${ }^{2-13}$ are particularly promising due to the availability of ultrashort laser pulses. However, the fundamental mechanisms of optically induced demagnetization and magnetic switching are not fully understood. In particular, the question of which reservoir supplies the angular momentum needed for demagnetization remains controversial. This reservoir plays a decisive role in determining the maximum demagnetization speed so resolving this issue is important for technological applications.

Most experiments on optical demagnetization and magneto-optical switching employ thermal methods. An optical pulse is absorbed, the electrons are driven far from equilibrium, and the sample is almost completely demagnetized within a few hundred femtoseconds. ${ }^{3-10}$ If this is performed in the presence of a magnetic field, the magnetization can be reversed. ${ }^{2-5}$

Some estimates show that thermal demagnetization occurs too rapidly for phonon processes to be relevant and it has been suggested that angular momentum is transferred between the spin and orbital components of the electrons. ${ }^{9}$ On the other hand, it is possible that the nonequilibrium electrons experience a spin-phonon interaction that is much stronger than usual. In this case, the phonons could provide the angular momentum. ${ }^{8,10}$ Transfer of angular momentum by the absorption of photons has also been considered. ${ }^{9,13,18}$ Theoretical arguments based on the number of photons absorbed ${ }^{9}$ and experiments using circularly polarized light with nickel ${ }^{18}$ indicate that the photon angular momentum is irrelevant, although experiments with GdFeCo yielded the opposite conclusion. ${ }^{13}$

Thermomagnetic switching is associated with an increase in temperature, so devices employing these methods will suffer a significant cooling time before new information can be written to them. The inverse Faraday effect (IFE) offers the possibility of nonthermally controlling the magnetization and avoiding this problem. ${ }^{11,12}$

The inverse Faraday effect is the generation of an effec- tive magnetic field using nonresonant circularly polarized light. ${ }^{19-21}$ It is the "inverse" of the well-known Faraday effect - the rotation of linearly polarized light propagating through a medium in a magnetic field ${ }^{22,23}$ _ because it is derived from the same free energy. ${ }^{19,20}$ The light is nonresonant, so photons are not absorbed and the temperature of the sample is unchanged.

In the classical IFE experiments, ${ }^{20,21}$ the light pulses had a width of $30 \mathrm{~ns}$. The slow variation in these pulses relative to the spin-lattice relaxation time ensured that the magnetization remained in thermodynamic equilibrium throughout, i.e., parallel to the effective magnetic field. The magnetic field provided an energy gradient, while the dynamics was caused by dissipation. On the other hand, the recent "ultrafast IFE" experiments ${ }^{11,12}$ used pulses that were only 100 $200 \mathrm{fs}$ in duration, and the magnetization dynamics was dominated by precession. Dissipation was only relevant for describing the subsequent return to equilibrium.

Magnetization reversal using the ultrafast IFE is therefore similar to precessional switching in an applied magnetic field, ${ }^{24-26}$ although the IFE has the advantage that optical pulses can be made much shorter than magnetic pulses. For short pulses, dissipation is known to be of minor importance ${ }^{26}$ and is neglected here.

Precession of the magnetization requires angular momentum. As with the thermomagnetic methods, the source of angular momentum in the IFE has not been identified despite much theoretical and experimental work. ${ }^{11-13,19-21}$ I show below that the angular momentum is provided by the photons. No other angular momentum reservoir is needed. This implies that the fundamental time scale of magnetic switching is limited only by our ability to find materials with a sufficiently strong magneto-optical response.

The near-equilibrium nature of the IFE simplifies the identification of the relevant angular momentum reservoir, as several of the above-mentioned options are eliminated. In the IFE, electrons are excited to higher orbital states, but these are virtual excitations and do not last long enough to make a significant contribution to the magnetization. In addition, precession occurs too rapidly and too close to equilibrium for phonons to be relevant.

It therefore appears that the photons are the only available source of angular momentum. However, it has been asserted that the angular momentum of the photons does not change because the IFE is caused by Raman scattering and both photon number and polarization are conserved. ${ }^{27}$ This con- 
tradiction is resolved by noting that spin-orbit coupling causes a spin-dependent change in propagation direction of the scattered photons, which changes their angular momentum. In classical terms, incident radiation is scattered anisotropically by a magnetic sample, so the maximum constructive interference between the scattered and incident light no longer occurs in the forward-scattering direction.

As in the classical derivation of the IFE, ${ }^{21} \mathrm{I}$ assume that the magnetic system is much smaller than the wavelength of the light so that the dipole approximation is appropriate. The extension to larger systems is briefly described at the end.

The spatial dependence of the spin is neglected and its magnitude is assumed constant. The spin is taken to be the only variable angular momentum of the system near the ground state. Finally, I assume that the amplitude of the laser pulse and the direction of the spin change adiabatically, i.e.,

$$
\left|\frac{d \epsilon_{i j}(\omega)}{d \omega}\right| \ll T\left|\epsilon_{i j}(\omega)\right|,
$$

where $\epsilon_{i j}(\omega)$ is the dielectric tensor, $\omega$ is the frequency of the light, and $T$ is the time scale on which the pulse changes or the spin precesses. ${ }^{28}$ Under this approximation, the light can be treated as a monochromatic plane wave and precession of the spin can be neglected when calculating the radiation field, as in the original derivation of the IFE. ${ }^{21}$ Note that the adiabatic condition is fulfilled even for ultrashort (50 fs) laser pulses if the frequency $\omega$ is more than $\sim 100 \mathrm{meV}$ from resonance.

If an infinite monochromatic plane wave were used in experiments, switching would not occur; the magnetization would simply precess until dissipation causes it to find the lowest energy state. Switching requires a finite, accurately tuned pulse, as for precessional switching with a magnetic field. ${ }^{29}$ However, if the adiabatic condition is fulfilled and dissipation is negligible, the time dependence of the pulse intensity does not influence the equations describing the IFE or angular momentum conservation. The time dependence of the pulse may be important when describing ultrafast IFE experiments using phase-modulated pulses ${ }^{27}$ unless the phase-modulated pulse can be described by a superposition of adiabatically varying laser pulses with different frequencies. In this case, the analysis presented below can be easily generalized.

Conservation of angular momentum for an electromagnetic field interacting with a material is described by ${ }^{30}$

$$
\frac{d}{d t} \int_{V}\left(\mathcal{L}_{\text {field }}+\mathcal{L}_{\text {mech }}\right)_{i} d V=-\epsilon_{i l m} \int_{S} T_{j l} r_{m} d S_{j} .
$$

Here, $\epsilon_{i l m}$ is the completely antisymmetric unit tensor of third rank, repeated indices are summed over, $\mathcal{L}_{\text {field }}$ and $\mathcal{L}_{\text {mech }}$ are the angular momentum densities for the electromagnetic field and the sample, respectively, $r_{m}$ is the position vector and $T_{j l}$ is the Maxwell stress tensor

$$
T_{j l}=\frac{1}{8 \pi}\left[2 E_{j} E_{l}+2 H_{j} H_{l}-\delta_{j l}\left(E^{2}+H^{2}\right)\right] .
$$

The integrals in Eq. (2) are taken over a large volume $V$ and the surface of this volume $S$.
The light is assumed nonresonant, so only motion within the ground-state manifold of the material is possible. For the small magnetic sample assumed here, the mechanical angular momentum $\int_{V} \mathcal{L}_{\text {mech }} d V$ can be replaced by the spin $\mathbf{S}(t)$.

The electric and magnetic fields entering Eq. (3) are superpositions of the homogeneous laser field

$$
\mathbf{E}(\mathbf{r}, t)=\frac{1}{2}\left(\mathbf{E}_{0} e^{i(\omega t-\mathbf{k} \cdot \mathbf{r})}+\text { c.c. }\right), \quad \mathbf{H}(\mathbf{r}, t)=\frac{\mathbf{k}}{k} \times \mathbf{E}(\mathbf{r}, t)
$$

and the radiation from the sample, which is treated as a dipole

$$
\mathbf{d}(t)=\frac{1}{2}\left\{\mathbf{d}_{0}[\omega, \mathbf{S}(t)] e^{i \omega t}+\text { c.c. }\right\} .
$$

Here, c.c. means complex conjugate and $\mathbf{d}_{0}[\omega, \mathbf{S}(t)]$ depends on the magnetization through spin-orbit coupling.

If the spin precession were nonadiabatic, the surface integral in Eq. (2) would be evaluated using the retarded dipole moment, $\mathbf{d}_{0}\left[\omega, \mathbf{S}\left(t^{\prime}\right)\right]$, where $t^{\prime}=t-R / c$ and $R$ is the distance between the sample and the surface $S$. All angular momentum emitted after time $t^{\prime}$ would not have reached the surface by time $t$. Instead of being measured as a flux across the surface $S$, this angular momentum would contribute to the volume integral

$$
\int_{V} \mathcal{L}_{\text {field }} d V \equiv \frac{1}{4 \pi c} \int_{V} \mathbf{r} \times(\mathbf{E} \times \mathbf{H}) d V .
$$

However, if the dynamics is adiabatic, retardation can be neglected and the surface integral in Eq. (2) can be evaluated using the "instantaneous" value of the dipole moment $\mathbf{d}_{0}[\omega, \mathbf{S}(t)]$. Then the integral in Eq. (6) becomes periodic with period $\pi / \omega$ and vanishes upon time averaging.

The surface integral in Eq. (2) is evaluated in the radiation limit $R \rightarrow \infty$ using Eqs. (3) and (4) and the classical expressions for the fields radiated by a dipole (see, e.g., Ref. 30). Equation (2) becomes

$$
\frac{d}{d t} \mathbf{S}(t)=\frac{1}{2} \operatorname{Re}\left\{\mathbf{d}_{0}[\omega, \mathbf{S}(t)] \times \mathbf{E}_{0}^{*}\right\}=\langle\mathbf{d}(t) \times \mathbf{E}(\mathbf{r}, t)\rangle_{\omega} .
$$

The brackets $\langle\cdots\rangle_{\omega}$ indicate time averaging over one period of the light and $\mathbf{E}_{0}^{*}$ is the complex conjugate of $\mathbf{E}_{0}$. The time averaging of $\mathbf{S}(t)$ and $\mathbf{d}_{0}[\omega, \mathbf{S}(t)]$ is neglected, as these quantities evolve adiabatically. Equation (7) can be rewritten in terms of experimentally measurable quantities by introducing the gyromagnetic ratio $\gamma$ and the magnetic moment $\mathbf{M}$ $=-|\gamma| \mathbf{S}($ Ref. 27):

$$
\frac{d}{d t} \mathbf{M}(t)=-\frac{|\gamma|}{2} \operatorname{Re}\left\{\mathbf{d}_{0}[\omega, \mathbf{S}(t)] \times \mathbf{E}_{0}^{*}\right\} .
$$

The right-hand side of Eq. (8) only contains terms linear in both $\mathbf{d}$ and $\mathbf{E}$. Terms quadratic in $\mathbf{d}$ have been neglected because induced fields are usually much weaker than incident fields. The term in $\mathbf{E}^{2}$ vanishes since homogeneous plane waves carry no angular momentum. ${ }^{31}$ Physically speaking, such a term would relate only to the incident light, not to the spin, and cannot appear in Eq. (8). The bilinearity of Eq. (8) in $\mathbf{E}$ and $\mathbf{d}$ indicates that the angular momentum 
gained by the spin is compensated by a change in the interference between the incident and scattered light.

The expression $\langle\mathbf{d}(t) \times \mathbf{E}(\mathbf{r}, t)\rangle_{\omega}$ in Eq. (8) is familiar as the time-averaged torque on a dipole in an oscillating electric field (see, e.g., Ref. 22). The appearance of this torque is not surprising, since the total angular momentum radiated by a dipole must be compensated by the torque on that dipole. ${ }^{32}$ The new feature described by Eq. (8) is that this electrically induced torque causes magnetization dynamics.

Equation (8) provides a clear understanding of how the inverse Faraday effect works: light induces a dipole moment, which is deflected from the plane in which the electric field oscillates by spin-orbit coupling. This deflection leads to a torque on the dipole moment by the light causing the spin to precess. This interpretation is a useful complement to the classical interpretation of the IFE-that spin flips occur due to the mixing of excited-state wave functions into the ground state. $^{21,27}$

If the torque is considered to be caused by an effective magnetic field then $d \mathbf{S} / d t=|\gamma| \mathbf{B}_{\text {eff }} \times \mathbf{S}$ (Ref. 27) and Eq. (7) becomes

$$
|\gamma| \mathbf{B}_{\mathrm{eff}} \times \mathbf{S}(t)=\frac{1}{2} \operatorname{Re}\left\{\mathbf{d}_{0}[\omega, \mathbf{S}(t)] \times \mathbf{E}_{0}^{*}\right\} .
$$

Equation (9) determines the components of $\mathbf{B}_{\text {eff }}$ orthogonal to $\mathbf{S}(t)$, i.e., the components that induce precession. The component of $\mathbf{B}_{\text {eff }}$ parallel to $\mathbf{S}(t)$ does not influence precession and cannot be recovered from Eq. (9), but it can be determined using conservation of energy.

The average energy of a dipole in an electric field is $U$ $=-\frac{1}{2}\langle\mathbf{d}(t) \cdot \mathbf{E}(\mathbf{r}, t)\rangle_{\omega}$ (see, e.g., Ref. 22 ; the factor $\frac{1}{2}$ arises because the dipole is induced by the field). This energy contains both a spin-dependent and a spin-independent contribution. The latter is removed by subtracting the spin-averaged dipole moment,

$$
\overline{\mathbf{d}}_{0}(\omega)=\int_{\mathcal{M}} \mathbf{d}_{0}(\omega, \mathbf{S}) d \mu(\mathbf{S})
$$

from $\mathbf{d}_{0}[\omega, \mathbf{S}(t)]$. Here, $\mathcal{M}$ is the space of admissible spin directions (i.e., the ground-state manifold) and $d \mu(\mathbf{S})$ is the appropriate normalized measure on this manifold. The result after subtraction is the Zeeman energy,

$$
|\gamma| \mathbf{B}_{\mathrm{eff}} \cdot \mathbf{S}(t)=-\frac{1}{4} \operatorname{Re}\left\{\left\{\mathbf{d}_{0}[\omega, \mathbf{S}(t)]-\overline{\mathbf{d}}_{0}(\omega)\right\} \cdot \mathbf{E}_{0}^{*}\right\} .
$$

As for Eq. (7), rapidly oscillating terms have been removed by averaging over one period of the light. From Eqs. (9) and (11), the effective magnetic field is

$$
|\gamma| \mathbf{B}_{\text {eff }}=\frac{1}{4|\mathbf{S}|^{2}} \operatorname{Re}\left\{2 \mathbf{S} \times\left(\mathbf{d}_{0} \times \mathbf{E}_{0}^{*}\right)-\mathbf{S}\left[\left(\mathbf{d}_{0}-\overline{\mathbf{d}}_{0}\right) \cdot \mathbf{E}_{0}^{*}\right]\right\} .
$$

Equation (12) seems to imply that $\mathbf{B}_{\text {eff }}$ depends on $\mathbf{S}(t)$. This would violate the general condition that an effective Hamiltonian cannot depend on the state of the sample within the ground-state manifold. I show now that Eq. (12) does not depend on $\mathbf{S}(t)$, at least for an isotropic system (isotropic except for magnetic order).

First, $\mathbf{d}_{0}$ is written in terms of the electric field and the polarizability tensor, $d_{i}=\alpha_{i j}(\mathbf{S}) E_{j}$, where repeated indices are summed over. The polarizability tensor can be expanded in a Taylor series in the spin:

$$
\alpha_{i j}=\alpha_{i j}^{(0)}+\alpha_{i j k}^{(0)} S_{k}+\cdots .
$$

The coefficients $\alpha_{i j}^{(0)}$ and $\alpha_{i j k}^{(0)}$ do not depend on the spin; the assumption of isotropy therefore implies that

$$
\alpha_{i j}^{(0)}=\lambda_{1} \delta_{i j} \text { and } \alpha_{i j k}^{(0)}=i \lambda_{2} \epsilon_{i j k} .
$$

If light is not absorbed, $\alpha_{i j}(\mathbf{S})$ must be Hermitian, so both $\lambda_{1}$ and $\lambda_{2}$ are real. Equation (12) becomes

$$
\mathbf{B}_{\text {eff }}=\frac{i \lambda_{2}}{4|\gamma|} \mathbf{E}_{0} \times \mathbf{E}_{0}^{*},
$$

which is the well-known expression for the IFE. ${ }^{21,27}$ The apparent dependence of $\mathbf{B}_{\text {eff }}$ on $\mathbf{S}$ has been removed.

To summarize: the magnetic field calculated from conservation of angular momentum is equal to the total IFE field. Precessional dynamics due to the IFE is fully compensated by angular momentum from the light. No other angular momentum reservoir is involved.

Equations (12) and (15) remain valid when $\mathbf{E}_{0}$ is replaced by a slowly varying $\mathbf{E}_{0}(t)$ that fulfills the adiabatic condition (1). This allows the modeling of pulses, which is essential for magnetic switching. It also allows Raman processes to occur. Rotating a spin in a real magnetic system requires a change in energy $\hbar \Delta \omega$, which must be supplied by the light. ${ }^{27}$ If we neglect spontaneous emission, rotation can only occur if the light contains modes with frequency $\omega-\Delta \omega$. Furthermore, because virtual transitions are instantaneous, the laser modes with frequencies $\omega-\Delta \omega$ and $\omega$ must be coherent. The replacement of $\mathbf{E}_{0}$ with $\mathbf{E}_{0}(t)$ allows these conditions to be fulfilled.

If rotating the spin requires energy, then as it departs from equilibrium, it will start to oscillate with the frequency $\Delta \omega$. The dipole radiation will then have the frequency $\omega-\Delta \omega$, despite being driven by an electric field with frequency $\omega$. When time averaged, the radiated light will exhibit no interference with the incident light except with the mode oscillating at the frequency $\omega-\Delta \omega$. Equation (15) becomes

$$
|\gamma| \mathbf{B}_{\mathrm{eff}}=\frac{i \lambda_{2}}{4} \mathbf{E}(\omega) \times \mathbf{E}^{*}(\omega-\Delta \omega) .
$$

For monochromatic light, $\mathbf{B}_{\text {eff }}=0$. The light may induce a change in spin via the terms in $\mathbf{d}^{2}$ that were neglected from Eq. (8), but this will be very weak. However, if the light has a sufficiently broad linewidth, Eq. (16) dominates the dynamics. This corresponds to the "stimulated Raman mechanism" mentioned in Ref. 21; the weaker "spontaneous Raman mechanism" cannot be investigated within the classical framework.

The above analysis uses the dipole approximation (5) and the assumption that $\mathbf{M}(t)$ has no spatial dependence. Both fail for extended systems, e.g., the experimental setup of 
Refs. 11 and 12, where the cross-sectional area of the magnetic sample is much larger than the laser spot. However, the fundamental mechanism of the IFE will not change- the angular momentum lost by each precessing spin is gained by the light. I briefly discuss this angular momentum transfer for extended systems, deferring a fuller discussion to a later paper.

Light in a solid cannot be separated into incident and scattered parts; all the light is coherently scattered. Usually, if the light beam enters at normal incidence, this scattering occurs in the forward direction and the beam propagates along a straight line. However, magnetization causes anisotropy in the coherent scattering, and the beam changes direction. (A similar effect occurs in anisotropic gases. ${ }^{34}$ ) While in the material, the beam propagates in the new direction. It emerges propagating parallel to its original direction of motion, but laterally displaced. This displacement is orthogonal to its linear momentum yielding a change in angular momentum.

For illustration, consider the experiment described in Ref. 12. Following Ref. 9, the number of illuminated spins is $N_{s}$ $\approx 4 \times 10^{19} A$, where $A$ is the area of the laser spot in $\mathrm{cm}^{2}$. The number of photons is $N_{p} \approx 4 \times 10^{16} A$. The magnetization changes by $\sim 1 \%$, which clearly cannot be achieved by changing the photon polarization. However, given a linear momentum of $2 \pi \hbar / \lambda$ per photon, with $\lambda=805 \mathrm{~nm}$, a deflection of the beam by $0.7 \mu \mathrm{m}$ would balance the angular momentum change in the magnetization. Such a deflection is well below a typical spot radius and is not easily visible. However, it is measurable in a carefully designed experiment, and would provide useful confirmation of the above results.

I have shown that in the inverse Faraday effect, the light both induces magnetization dynamics and provides the necessary angular momentum; no other source of angular momentum is required. If transparent magnetic materials can be found with a sufficiently strong magneto-optical response, full magnetization reversal could occur on the time scale of a laser pulse.

S.R.W. thanks R. O. Jones and A. Bringer for many useful suggestions and help in preparing this Brief Report. I also thank A. Liebsch and S. Blügel for valuable discussions. *simon.woodford@icr.ac.uk

${ }^{1}$ I. Tudosa et al., Nature (London) 428, 831 (2004).

${ }^{2}$ M. H. Kryder, J. Appl. Phys. 57, 3913 (1985).

${ }^{3}$ D. Guarisco, R. Burgermeister, C. Stamm, and F. Meier, Appl. Phys. Lett. 68, 1729 (1996).

${ }^{4}$ G. P. Ju, L. Chen, A. V. Nurmikko, R. F. C. Farrow, R. F. Marks, M. J. Carey, and B. A. Gurney, Phys. Rev. B 62, 1171 (2000).

${ }^{5}$ J. Hohlfeld, T. Gerrits, M. Bilderbeek, T. Rasing, H. Awano, and N. Ohta, Phys. Rev. B 65, 012413 (2001).

${ }^{6}$ E. Beaurepaire, J. C. Merle, A. Daunois, and J. Y. Bigot, Phys. Rev. Lett. 76, 4250 (1996).

${ }^{7}$ J. Hohlfeld, E. Matthias, R. Knorren, and K. H. Bennemann, Phys. Rev. Lett. 78, 4861 (1997).

${ }^{8}$ B. Koopmans, M. van Kampen, J. T. Kohlhepp, and W. J. M. de Jonge, Phys. Rev. Lett. 85, 844 (2000).

${ }^{9}$ B. Koopmans, M. van Kampen, and W. J. M. de Jonge, J. Phys.: Condens. Matter 15, S723 (2003).

${ }^{10}$ C. Stamm et al., Nature Mater. 6, 740 (2007).

${ }^{11}$ A. V. Kimel et al., Nature (London) 435, 655 (2005).

${ }^{12}$ F. Hansteen, A. Kimel, A. Kirilyuk, and T. Rasing, Phys. Rev. B 73, 014421 (2006).

${ }^{13}$ C. D. Stanciu, F. Hansteen, A. V. Kimel, A. Kirilyuk, A. Tsukamoto, A. Itoh, and T. Rasing, Phys. Rev. Lett. 99, 047601 (2007).

${ }^{14}$ J. C. Slonczewski, J. Magn. Magn. Mater. 159, L1 (1996).

${ }^{15}$ A. Asamitsu et al., Nature (London) 388, 50 (1997).

${ }^{16} \mathrm{H}$. Ohno et al., Nature (London) 408, 944 (2000).

${ }^{17}$ Y. Kato et al., Nature (London) 427, 50 (2004).

${ }^{18}$ F. Dalla Longa, J. T. Kohlhepp, W. J. M. de Jonge, and B. Koopmans, Phys. Rev. B 75, 224431 (2007).

${ }^{19}$ P. S. Pershan, Phys. Rev. 130, 919 (1963).
${ }^{20}$ J. P. van der Ziel, P. S. Pershan, and L. D. Malmstrom, Phys. Rev. Lett. 15, 190 (1965).

${ }^{21}$ P. S. Pershan, J. P. van der Ziel, and L. D. Malmstrom, Phys. Rev. 143, 574 (1966).

${ }^{22}$ L. D. Landau and E. M. Lifshitz, Electrodynamics of Continuous Media (Pergamon Press, Oxford, 1960).

${ }^{23}$ A. D. Buckingham and P. J. Stephens, Annu. Rev. Phys. Chem. 17, 399 (1966).

${ }^{24}$ C. H. Back et al., Science 285, 864 (1999).

${ }^{25}$ C. H. Back, D. Weller, J. Heidmann, D. Mauri, D. Guarisco, E. L. Garwin, and H. C. Siegmann, Phys. Rev. Lett. 81, 3251 (1998).

${ }^{26}$ M. Bauer, J. Fassbender, B. Hillebrands, and R. L. Stamps, Phys. Rev. B 61, 3410 (2000).

${ }^{27}$ A. V. Kimel, A. Kirilyuk, and Th. Rasing, Laser Photonics Rev. 1, 275 (2007).

${ }^{28}$ L. P. Pitaevskii, Sov. Phys. JETP 12, 1008 (1961).

${ }^{29}$ Th. Gerrits et al., Nature (London) 418, 509 (2002).

${ }^{30}$ J. D. Jackson, Classical Electrodynamics (John \& Wiley, New York, 1962).

${ }^{31}$ I. V. Sokolov, Sov. Phys. Usp. 34, 925 (1991).

${ }^{32}$ This is a very general statement, since torque and change in angular momentum are related by definition. However, the explicit demonstration that the torque on a dipole in an electric field is equal (and opposite to) the radiation of angular momentum from the dipole was only demonstrated less than 30 years ago, in Ref. 33 .

${ }^{33}$ A. P. Kovalenko and A. A. Kolokolov, Radiophys. Quantum Electron. 25, 283 (1982).

${ }^{34}$ G. Moe and W. Happer, J. Phys. B 10, 1191 (1977). 